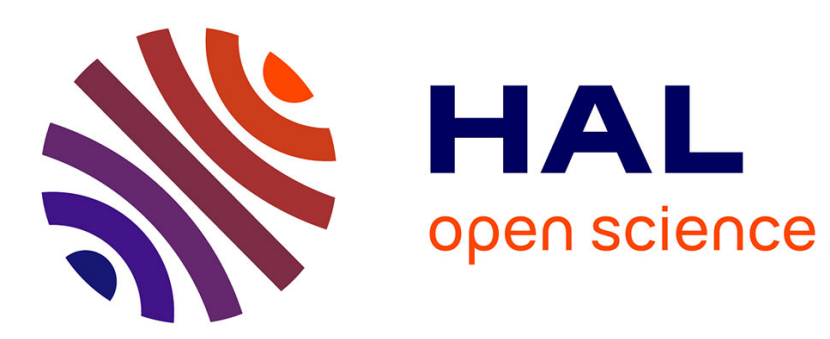

\title{
Relations de travail dans le Laos rural : économie morale paysanne et éthique de l'entrepreneur
}

\author{
Vanina Bouté
}

\section{To cite this version:}

Vanina Bouté. Relations de travail dans le Laos rural : économie morale paysanne et éthique de l'entrepreneur. Autrepart - Revue de sciences sociales au Sud, 2017, N83 (3), pp. 89-104. 10.3917/autr.083.0089 . hal-02478834

\section{HAL Id: hal-02478834 \\ https://hal.science/hal-02478834}

Submitted on 20 Dec 2020

HAL is a multi-disciplinary open access archive for the deposit and dissemination of scientific research documents, whether they are published or not. The documents may come from teaching and research institutions in France or abroad, or from public or private research centers.
L'archive ouverte pluridisciplinaire HAL, est destinée au dépôt et à la diffusion de documents scientifiques de niveau recherche, publiés ou non, émanant des établissements d'enseignement et de recherche français ou étrangers, des laboratoires publics ou privés. 


\title{
RELATIONS DE TRAVAIL DANS LE LAOS RURAL : ÉCONOMIE MORALE PAYSANNE ET ÉTHIQUE DE L'ENTREPRENEUR
}

Vanina Bouté

\author{
Presses de Sciences Po | «Autrepart»
}

2017/3 N 83 | pages 89 à 104

ISSN 1278-3986

ISBN 9782724635362

Article disponible en ligne à l'adresse :

https://www.cairn.info/revue-autrepart-2017-3-page-89.htm

Distribution électronique Cairn.info pour Presses de Sciences Po.

(C) Presses de Sciences Po. Tous droits réservés pour tous pays.

La reproduction ou représentation de cet article, notamment par photocopie, n'est autorisée que dans les limites des conditions générales d'utilisation du site ou, le cas échéant, des conditions générales de la licence souscrite par votre établissement. Toute autre reproduction ou représentation, en tout ou partie, sous quelque forme et de quelque manière que ce soit, est interdite sauf accord préalable et écrit de l'éditeur, en dehors des cas prévus par la législation en vigueur en France. Il est précisé que son stockage dans une base de données est également interdit. 


\title{
Relations de travail dans le Laos rural : économie morale paysanne et éthique de l'entrepreneur
}

\author{
Vanina Bouté*
}

Il n'existe pas d'études sur l'apparition du travail salarié dans le monde paysan au Laos. Pourtant, de façon croissante depuis ces dix dernières années, les riziculteurs sont fortement incités à abandonner une économie d'autosuffisance pour passer à la production de cultures commerciales pour des entrepreneurs laotiens et surtout, pour les concessions chinoises qui se multiplient dans le nord du pays. Quelques chercheurs ont mentionné le fait que les entreprises rémunèrent mal, voire pas du tout, les paysans qu'elles emploient, ou qu'il n'existe pas de cadre légal à l'embauche [Baird, 2010 ; Rigg, 2005]. Un article récent de Li et Littleton [2017] explore toutefois comment la notion de Guanxi, soit une culture de relations personnelles à travers l'échange de cadeaux, de faveurs et de banquets, est utilisée par les entrepreneurs chinois pour consolider leurs transactions économiques avec des paysans Akha du nord du Laos. Même si les auteurs ne le mentionnent pas, ce qu'ils appellent « la dimension affective des échanges » fait irrésistiblement penser au concept «d'économie morale » développé tout d'abord par Edward Thompson pour les classes populaires en Angleterre [1971], puis repris par James Scott [1976] pour expliquer les révoltes paysannes en Birmanie et au Vietnam. Chez Scott, comme chez Thompson, « l'économie morale » renvoie à une conception des relations et des transactions économiques entre le peuple et les élites, qui sont indissociables des normes sociales, des valeurs et des obligations inscrites dans les routines quotidiennes.

L'objectif de cet article est de s'interroger sur l'existence, et surtout la nature, de normes et de valeurs dans les relations de travail entre employeurs et employés, paysans et entrepreneurs laotiens et chinois dans le nord du Laos. Ces relations sont en effet nouvelles. Les paysans qui deviennent des travailleurs salariés sont originaires de zones de montagnes. Ils ont migré, non mus par des considérations économiques, mais par la nécessité de reformer des réseaux familiaux après la disparition, souvent programmée par l'État, de leur village d'origine [Bouté, 2014].

1. Département de sociologie et d'ethnologie, université de Picardie, membre du Centre Asie du Sud-Est (UMR 8170, CNRS-EHESS-Inalco). 
La plupart d'entre eux imaginaient pouvoir reprendre dans les basses terres une activité agricole tournée vers l'autosuffisance, comme cela était le cas dans leur village d'origine. Mais face aux difficultés d'accéder à des terres dans les localités d'accueil, la plupart finissent par accepter des emplois journaliers. Alors que les relations de travail dans leur village d'origine étaient avant tout des relations horizontales, de coopération et d'entraide, on peut s'interroger sur les modalités du passage au travail salarié, et surtout sur les représentations qui y sont désormais associées.

À l'instar d'autres chercheurs [Bernal, 1994 ; Roitman, 2000], je considérerai que ce n'est pas parce qu'il y a intégration dans un système capitaliste, qu'il y a renoncement complet à certaines valeurs «non capitalistes ». De fait, à travail égal, les paysans préfèrent se tourner non pas vers un patron qui les rémunère mieux, mais vers celui qu'ils définissent comme un «bon patron »; tandis que tout l'enjeu, pour ces derniers, est de retenir et de captiver une main-d'œuvre a priori volatile. C'est donc les attentes des uns, les usages des autres et leur façon d'envisager mutuellement leurs relations de travail que nous examinerons à travers quelques exemples tirés d'enquêtes ethnographiques menées dans le district de Boun Tay, dans la province de Phongsaly, la plus septentrionale du Laos ${ }^{1}$.

\section{De l'agriculture de montagne au salariat dans les plaines : un monde rural en plein bouleversement}

Le Laos est le seul pays d'Asie du Sud-Est sans accès à la mer. Avec six millions d'habitants, il est très peu peuplé (surtout au regard de ses voisins), et essentiellement montagneux. Jusque dans les années 2000, sa population était à $80 \%$ rurale. Sur les quelques terres planes du pays, les plaines du long du Mékong et les fonds de vallées, se trouvaient majoritairement les populations de langue lao-taï (qui forment à peu près $60 \%$ de la population du pays) vivant de la riziculture en casiers. Les terres montagneuses étaient occupées par les $40 \%$ de la population parlant des langues tibéto-birmanes, môn-khmères, hmong-mien, qui vivaient essentiellement de culture du riz sur brûlis, et d'un peu d'élevage. Les pratiques agricoles des habitants des villages sont presque entièrement tournées vers l'autosuffisance ${ }^{2}$. La valeur essentielle est le riz qui est le principal indicateur de richesse au sein des foyers. Le travail (asip) est alors essentiellement considéré comme devant être celui du paysan, riziculteur ou essarteur (saona, saohay), ou du fonctionnaire (latakone). L'importance accordée par la République démocratique populaire lao au monde paysan était mise en avant à travers l'imagerie des

2. Cet article s'appuie sur des enquêtes ethnographiques de plusieurs, mois menées dans le nord du Laos (provinces d'Oudomxay, Luang Namtha et surtout Phongsaly) entre 2012 et 2015, dans le cadre du programme de recherche européen FP7 SEATIDE - Southeast Asia : Trajectories of inclusion, dynamics of exclusion. Je remercie ici les deux relecteurs de Autrepart pour leurs commentaires stimulants sur ce papier.

3. En dehors de la production familiale d'opium dans les essarts de montagne en hiver. L'opium était cultivé légalement jusqu'en 2002 (le gouvernement prélevait officiellement des taxes sur la production). Depuis 2005, la production est interdite. 
panneaux de propagande, des billets de banque, des timbres, des statues. Les uns donnaient à lire l'unité d'une nation pluriethnique à travers trois femmes d'ethnies différentes, représentées comme trois paysannes, faucille dans une main, gerbe de riz dans l'autre. D'autres célébraient l'union des travailleurs à travers les figures du fonctionnaire, du paysan, et du soldat.

Dans les années 1990, le modèle économique de développement préconisé (tant par le gouvernement que par les bailleurs internationaux comme le FMI, la Banque mondiale, ou la Banque asiatique de développement) repose sur un modèle d'agriculture supposé «moderne », celui pratiqué par les Lao, l'ethnie majoritaire, qui est la riziculture inondée, et induit la condamnation de la défriche-brûlis [Évrard, Baird, 2017]. La riziculture inondée étant difficilement réalisable en zone montagneuse, des déplacements de populations d'essarteurs furent organisés à l'échelle nationale, entraînant des relocalisations massives de villages transférés dans les plaines ou le long des routes [Goudineau, 1997]. Dans les années 2000, après l'entrée du Laos au sein de l'Asean (Association of Southeast Asian Nations), et dans le contexte d'une intégration régionale et globale accrue, à la riziculture en plaine s'ajouta la mise en avant de la production de cultures commercialisables. Les déplacements de villages en bord de route se poursuivirent afin de faciliter la production de ces cultures (thé, hévéa, café, maïs, etc.), initiées par des investisseurs étrangers (principalement chinois dans le nord du pays); d'autres villages furent, et continuent d'être évacués pour permettre l'aménagement d'infrastructures de taille importante (barrages, mines, concessions), souvent liées à des investisseurs étrangers [Dwyer, 2007].

On peut ajouter à ce tableau un troisième phénomène important apparu depuis 2010 (et, à ma connaissance, très peu documenté) qui, à la différence des deux premiers, ne résulte pas d'une réforme promue par le gouvernement et ne concerne pas les populations des montagnes, à savoir le développement des cultures commerciales dans les rizières en saison sèche. Ce nouveau marché est pris en charge et promu par de petites entreprises chinoises basées dans la province du Yunnan. Elles s'installent dans les provinces frontalières du nord du Laos le temps d'une saison ou de façon permanente, en établissant un partenariat avec un Laotien pour l'obtention d'autorisations administratives. Le marché consiste à proposer des semences, des intrants et des engrais aux paysans, qui fournissent en échange la terre et la main-d'œuvre (un contrat bien connu sous le terme « $2+3$ » [Dwyer ; 2007]). Les cultures commerciales - jusqu'à présent principalement des piments et des haricots verts-sont plantées en saison sèche lorsque les rizières sont inutilisées, ce qui transforme tout agriculteur en producteur pour ce marché à partir du moment où il est propriétaire de sa terre.

Je prendrai comme illustration de ce processus Boun Tay, le district de la province de Phongsaly, au nord du Laos, qui connaît aujourd'hui le développement le plus spectaculaire de la province ${ }^{3}$. À l'époque de sa fondation en 1993, son

4. C'est là où j'ai mené l'enquête en janvier-février 2015 sur la thématique du présent article, mais j'y avais travaillé à plusieurs reprises depuis 2005, en particulier sur le thème de la migration. La province 
chef-lieu, situé dans une petite cuvette rizicole bordée de montagnes, comportait une quarantaine de fonctionnaires et leurs familles installés à proximité d'un village de riziculteurs tai lü, Boun Tay. Très rapidement, cette localité attira de nombreuses familles issues des villages de montagnes environnants et de districts voisins. Ils s'installèrent en mettant en valeur des terres non exploitées, soit les surfaces de rizières vacantes ou les friches aménageables en rizières qui étaient disponibles jusqu'en 1998. En une vingtaine d'années, l'accroissement démographique du district fut spectaculaire : de 10937 personnes en 1993, la population passa à environ 15200 en 2003, et à 23100 dix ans plus tard ${ }^{4}$.

À partir de 2007, la culture de l'hévéa se répandit dans les fonds de vallée. Ce fut d'abord le fait d'entreprises chinoises qui établirent rapidement des concessions et prirent possession des plus gros lopins. Elles furent rapidement imitées par des commerçants et des fonctionnaires du district, mais surtout de la capitale de province, située à une centaine de kilomètres de là, qui achetèrent des terres et firent planter de l'hévéa. Deux années après, le mouvement devint général et tous les habitants du chef-lieu qui en avaient les moyens plantèrent à leur tour de l'hévéa sur leurs terres à essarts. Se développa alors de façon exponentielle le besoin de main-d'œuvre salariée. Pour s'occuper de leurs plantations, les plus gros propriétaires emploient le plus souvent des paysans sans terres issus des alentours. Plus rarement, ils font venir des familles des villages de montagnes. Très peu payés, ces employés vivent essentiellement du riz et des légumes qu'ils cultivent entre les plants d'hévéa.

Ce recentrement des populations vers les basses terres et les villes (et, en montagne, sur les bords de route) a abouti à une certaine pression foncière [Bouté, 2014]. Le corollaire de cette pression foncière est la marchandisation de la terre, au point que certains parlent aujourd'hui «d'or vert », face aux demandes accrues pour transformer les terres en plantations [Dwyer, 2007]. À partir des années 2010, les terres cultivables ont commencé à se faire rares, et les familles (environ $15 \%$ ) installées depuis cette époque n'ont pas de rizières : elles louent des terres d'essarts pour y cultiver du riz. Ceci a ralenti le flux migratoire, et l'a progressivement réservé à ceux qui possédaient déjà un réseau de parents en plaine, capables de les faire venir ${ }^{5}$.

Lorsque les prix du caoutchouc s'effondrent quelques années après, le besoin de main-d'œuvre perdure grâce au développement très rapide de la culture des haricots par de petites entreprises chinoises dans les rizières en saison sèche. La

de Phongsaly est la plus septentrionale du Laos, bordée par la Chine, et par le Vietnam. Elle est essentiellement montagneuse, avec des fonds de vallée encaissés où vivent des piroguiers lao ou des riziculteurs de langue taï (Tai Lü, Dam, Deng), et dans les montagnes, des populations de langue austro-asiatique (Khmou, Bit), tibéto-birmane (Phounoy, Akha) et hmong-mien (Hmong, Yao).

5. Soit une augmentation de $39 \%$ entre 1993 et 2003, et de $52 \%$ entre 2003 et 2013 - des chiffres bien supérieurs à la moyenne nationale $(22 \%$ et de $19 \%$ pour ces deux périodes) et à celle de la province de Phongsaly $(8 \%$ et $4 \%$ ) [Steering committee for census of population and housing, $2006 ; 2016$ ].

6. Entre 2013 et 2014 , le taux d'accroissement annuel de la population du district n'a été que de $1,2 \%$ (contre $2,1 \%$ au niveau national). 
raison de ce succès est double. Cette culture a lieu après la récolte du riz qui était jusque-là une période de chômage agricole et de vacance de la terre. De plus, cette culture est très lucrative : stimulée par les engrais fournis par les entreprises, une surface de rizière qui peine à assurer la suffisance en riz de deux personnes peut offrir deux à trois tonnes de haricot en quatre mois, soit plus de 10 millions de kips (1 240 US \$), presque le salaire annuel d'un fonctionnaire.

La récolte de ces haricots réclame une importante main-d'œuvre : en plus des traducteurs qui puissent faire le lien entre paysans et patrons chinois, des conducteurs de camions qui rapportent quotidiennement les haricots en Chine, et des contremaîtres, le travail repose essentiellement sur ceux qui cueillent et mettent en caisse les haricots, en l'occurrence une main-d'œuvre flottante qui vient quotidiennement se proposer à l'entreprise. Notons enfin que le développement d'une bourgade avec la construction de bâtiments, d'hôtels, de restaurants et, maisons privées a créé aussi des emplois non agricoles, tels que femmes de ménage, gardiens, maçons et chefs de chantier.

Le développement de l'hévéa et des cultures commerciales dans les rizières, ainsi que la demande de services liée à l'enrichissement de certains producteurs, ont entraîné une forte demande de main-d'œuvre salariée, mais le ralentissement des migrations paysannes vers Boun Tay, en raison de la pression foncière que j'évoquais précédemment, fait que la main-d'œuvre disponible manque. Un fonctionnaire de l'hôpital déplorait : «Il n'y a pas beaucoup de gens qui cherchent un travail salarié, car ils n'ont pas de temps. À peine le riz est récolté, les Chinois leur demandent de planter les haricots ». Un jeune paysan tai lü se plaignait, lui, de ne trouver personne pour l'aider à récolter : «Avec ma belle-famille, nous avons récolté presque cinq tonnes; on pourrait obtenir encore plus, mais le problème est que tout le monde cultive des haricots et du coup, il n'y a personne pour venir travailler pour nous ». La propriétaire d'une des guest houses de la ville racontait elle aussi : «Les employés pour l'hôtel ? Ils viennent un instant puis repartent du jour au lendemain. Je suis fatiguée de chercher ».

Les propos tels que ceux tenus par la tenancière d'hôtel déplorant une maind'œuvre volatile laissent penser que, les journaliers étant moins nombreux que les postes à fournir, ils trouvent aisément du travail et peuvent en changer facilement. Mais la situation s'avère plus complexe : car ce qu'on entend localement par « un travail », ou plutôt par « un bon travail » exclut un certain nombre d'emplois. Il apparaît que ce n'est ni la nature du travail ni le salaire qui le définissent, mais la nature des interactions entre employeurs et salariés.

\section{Les paysans salariés : un changement de la notion de réciprocité}

Dans son étude du Laos socialiste des années 1980, l'anthropologue Grant Evans [1990] s'intéressait à la mise en place, par le gouvernement révolutionnaire, de programmes de collectivisation visant à remplacer l'économie d'autosuffisance (natural économy) des paysans; l'argument principal de Evans était que le 
gouvernement avait finalement dû renoncer à ces programmes, car les paysans y avaient opposé une forte résistance, notamment parce qu'ils étaient contraires à la division et à la rémunération traditionnelles du travail, à l'échange de services, aux rôles des femmes et des enfants dans la production ainsi qu'aux relations intergénérationnelles au sein de la famille et du village. Evans [1990, p. 30] notait que cette économie morale qui imprégnait le monde paysan valait ailleurs et que les relations entre élites urbaines émergentes et paysans, quoique distantes, étaient appréciées, de façon similaire, à l'aune de règles de réciprocité.

Dans un contexte où la production de cultures commerciales et l'embauche de paysans comme journaliers apparaissent a priori comme des indicateurs du passage à une économie capitaliste, on peut s'interroger sur le devenir des relations de coopération entre paysans, ainsi qu'aux relations entre paysans et élites locales. C'est donc non aux dimensions économiques du travail que nous allons nous intéresser ici, mais aux formes de représentations qui y sont associées.

Le fait de travailler pour les autres, ainsi que les termes pour qualifier ce travail ont changé entre 2005 et 2015. Lors de mes premiers séjours au début des années 2000, le terme asip était employé indifféremment par les paysans et les fonctionnaires pour désigner leur principale occupation (faire la rizière, cultiver des essarts, travailler pour le gouvernement). Dix ans plus tard, asip désigne uniquement une occupation rémunérée. Ce qui faisait dire à certains fonctionnaires que les montagnards vivant d'agriculture d'autosuffisance n'avaient pas d'occupation (asip). Le travail journalier, lui, est désigné par le terme tjang kin (littéralement «embaucher-manger», que l'on pourrait traduire par «travail alimentaire »). Il est rarement considéré comme une occupation durable, avec pour argument qu'il est trop dépendant de la force physique (on ne peut plus l'exercer avec l'âge) ou trop aléatoire (ce qu'on gagne en un jour, on le «mange» le lendemain).

Les représentations liées au travail pour les autres ont également changé au cours de la dernière décennie. Lors de mon premier séjour il y a 15 ans, les paysans pratiquaient l'échange de journées de travail et allaient travailler dans les champs les uns des autres, surtout en période de semis. Cette forme de coopération basée sur la réciprocité, qualifiée «d'entraide» (souaykan), était perçue comme une manière de faire évidente, une nécessité morale. Comme le notait Grant Evans [1990, p. 212] dans les années 1990 dans la plaine de Vientiane, alors que l'échange de travail prenait place entre égaux et pouvait entraîner des compromis, le travail salarié était considéré comme le fait d'être à la merci de quelqu'un.

Les premières expériences du travail salarié semblent avoir suscité de ce fait des réactions douloureuses. La sœur de Souk, installée depuis vingt ans à Boun Tay, travaille à présent comme femme de ménage dans un hôtel chinois récemment construit; elle se souvient qu'il y a quelques années, elle refusait d'aller travailler chez les autres : «j'avais honte (na ay) et j'avais peur (yan)», dit-elle. Singvane, originaire d'un village de montagne de Phongsaly est arrivée avec son mari à Boun Tay en 2005, à la suite de la disparition de son village, 
dont toute la population avait migré vers les basses terres. Elle fut sollicitée pour effectuer un travail rémunéré mais son mari s'y opposa fermement, en dépit du fait qu'ils vivaient dans des conditions précaires; il avait honte (na ay) que sa femme travaille pour des étrangers. Oun, originaire du même village s'est installée à la périphérie de la ville de Phongsaly à la même époque. Le champ de théiers qu'elle et son mari avaient acheté ne suffisait pas à leurs besoins et son conjoint a commencé à travailler comme forgeron. Quant à Oun, alors âgée de 50 ans, elle a trouvé un travail de cantinière pour une entreprise vietnamienne qui venait goudronner la route. «Tous les jours, je pleurais, et pleurais tellement j'avais honte de travailler chez d'autres gens », raconte-t-elle.

Pourtant, lorsque j'interrogeais plus avant les paysans sur cette honte, les raisons énoncées n'étaient plus tant relatives au problème d'être au service de quelqu'un d'autre, qu'au souci de ne pas savoir comment se comporter dans une situation nouvelle : «On a honte de ne pas être capable de faire le travail au début parce qu'on ne le connaît pas »; «On a honte parce qu'on n'a pas l'habitude d'être avec des gens nouveaux qui voient qu'on n'est pas à l'aise »; «La honte, c'est de ne pas savoir comment s'exprimer, ne pas oser parce qu'on est de petites gens (phou noy) ». L'inconfort ressenti par la plupart des paysans passant au travail salarié traduit certes celui lié au passage de relations horizontales (celles d'entraide que pouvaient avoir les paysans au sein d'un même village ou entre villages voisins) à des relations verticales. Mais ce n'est pas tant le passage à une relation contractuelle qui semble poser problème que l'incapacité à maîtriser les codes requis dans une situation nouvelle.

Cette maîtrise des codes requis lors de nouvelles interactions est jugée d'autant plus importante qu'elle permet d'établir de nouvelles formes de réciprocité qui doivent, pour être comprises comme telles, être validées par chacune des parties en présence. Avant de développer plus avant ce qu'employés et employeurs conçoivent devoir être les formes de réciprocité appropriées à leur relation, disons déjà que la réciprocité au Laos est une notion essentielle dans l'échange, quelle que soit sa nature, car elle permet des deux côtés de ne pas perdre (sia) la face (na) ou l'estime de soi (piep) ${ }^{6}$. Dans son essai sur la notion d'autorité traditionnelle au Laos, Amphay Doré définissait le piep comme le patrimoine vital de l'individu au Laos, patrimoine qui s'appauvrit ou s'accroît dans les échanges entre individus ou groupes : «On conçoit que le piep soit considéré comme la chose la plus précieuse pour les Lao : si l'on ne sait le garder (vay piep), on est déclassé (tok san tam) » [Doré, 1972, p. 26]. C'est ainsi que l'absence de réciprocité dans l'échange entraîne une perte de piep ou de face (na) selon les contextes, qui entraîne à son tour la honte.

7. La notion de piep, étroitement associée à celle de face (na) est définie dans le dictionnaire de Marc Reinhorn [1970] comme « l'honneur, la personnalité, la renommée, la considération ». Inversement, «perdre du piep » serait «se déconsidérer». 
La crainte d'une absence de réciprocité peut naître d'interactions nouvelles dont on ne maîtrise pas encore les modalités, mais aussi de la transformation des anciennes formes d'échanges qui se révèlent obsolètes dans ce contexte de changement rapide. Dix ans plus tard, lorsque je les réinterroge, ceux qui me disaient avoir honte maîtrisent à présent certains codes et savent où travailler pour ne pas ressentir de honte : la sœur de Souk dit s'être habituée à son emploi à l'hôtel ; Singvane et son mari n'ont pas de terres, mais trouvent tous les jours à s'employer, elle, pour la cueillette de haricots, lui, comme maçon, etc. Tous estiment que la honte liée au travail salarié est un signe d'inadaptation, car désormais leur subsistance dépend de nouvelles formes de transaction.

Inversement, les anciennes formes d'échanges ne semblent plus être comprises comme telles dans ces nouveaux contextes. L'échange réciproque de travail, autrefois courant pour certaines opérations (semailles, battage, réparation des maisons), est devenu rare, ou restreint au seul cercle familial. De même, les échanges de biens entre non-parents, qui étaient si fréquents dans les villages de Phongsaly lors de mes premiers séjours au début des années 2000, sont devenus inexistants : ils permettaient pourtant d'obtenir de l'alcool de riz contre un cochon, du riz contre des produits forestiers, du gibier contre une journée de travail, etc. Comme le note Holly High [2008, p. 535] pour le sud du Laos, certains paysans qui y ont encore recours le font parfois à contrecœur, assimilant leur démarche à de la mendicité. Dans ce cas, c'est bien, à mon sens, parce que la notion de services réciproques ne semble plus aller de soi ou être comprise des deux côtés que l'échange n'est plus possible sans faire intervenir la honte.

Dans le contexte, nouveau pour le nord du Laos, d'une généralisation des relations de travail verticales, ce qui est conçu comme « réciprocité »n'est pas mesuré à l'aune du salaire versé contre la force de travail. S'assurer du recrutement et du maintien d'une main-d'œuvre extrêmement volatile, car non contrainte, dans un contexte où, on l'a vu, l'offre de travail est largement supérieure à la demande implique donc pour un employeur ou un entrepreneur de mettre en jeu autre chose que le simple salariat, soit certaines formes d'engagements conformes aux attentes de ses employés. D'un autre côté, les paysans m'affirmaient qu'il était difficile de trouver du travail, car il ne leur était pas possible de travailler pour n'importe qui.

L'un des critères pour arriver à recruter, ou à engager des paysans pour une opération contractuelle, est le don, matériel, dans un premier temps. Je ne reviendrai pas ici sur une discussion autour de la notion de don, ${ }^{7}$ mais disons juste qu'il s'agit de situations où le don ou la promesse de don est nécessaire pour engager une relation contractuelle entre les deux parties. Si le travail à effectuer est souvent le même, et si le profil des employeurs varie (patrons d'entreprises chinoises, contremaîtres de plantations, fonctionnaires locaux, commerçants, autres paysans), cette notion du don nécessaire et préalable à tout engagement dans une relation

8. Voir par exemple Mariani [2012] autour d'opérations de dons au Laos dans la capitale, Vientiane. 
semble être comprise par beaucoup. Un entrepreneur lao de Boun Tay, Khamman, cherchait ainsi à convaincre les paysans du village de Bonoy de planter des haricots pour pouvoir ensuite les lui revendre. Ces derniers, des riziculteurs aisés, refusaient obstinément ses propositions ainsi que celles d'entreprises chinoises concurrentes. Il finit par remporter leur adhésion en faisant construire à ses frais une maison commune destinée aux réunions administratives du village. Lorsque je l'interroge, il raconte : «J'ai fait cela pour que les gens ne doutent pas de moi ». Inversement, le manquement à la promesse d'un don suffit à rompre toute forme d'engagement comme l'illustre l'exemple du village de Hatngam. Les habitants de ce village s'étaient déplacés depuis la montagne jusqu'à un fond de vallée, à l'instigation d'une entreprise chinoise qui leur avait proposé de venir travailler dans sa concession pour planter, puis saigner des hévéas. Sans s'attarder sur les péripéties de leur contrat, disons que les paysans estimaient avoir été floués par l'entreprise qui leur avait promis, en sus d'un travail salarié, une aide matérielle en contrepartie de leur déménagement (tôles pour les toits des maisons, outils, semences, etc.) et n'avait pas tenu cet engagement. Sept ans plus tard, alors que les arbres plantés par les paysans sur la concession étaient arrivés à maturité, et que la récolte du caoutchouc devenait possible, ces derniers refusèrent de saigner les arbres contre salaire, alors même que certains d'entre eux recherchaient des emplois équivalents à plusieurs kilomètres de là et que les autres se plaignaient, étant éloignés du chef-lieu du district, de ne pas avoir d'opportunité d'accéder au travail salarié. Mes interlocuteurs m'expliquèrent ce refus par un manquement de l'entreprise à leur égard : « S'ils s'étaient occupés de nous, on irait, mais ils n'ont rien fait pour nous ; ils n'ont pas tenu leurs engagements ». Ici, c'est la valeur de la parole donnée qui est essentielle ; la rupture de l'engagement donné suffit aux paysans pour refuser toute nouvelle collaboration.

Si le salaire n'est donc pas une condition suffisante pour accepter un travail, le don matériel ne permet pas seul d'engager ou de maintenir des relations de réciprocité. Au terme de leur analyse de «l'affectivité des échanges » entre entrepreneurs chinois et paysans Akha dans le nord du Laos, Li et Lyttleton [2017, p. 321] concluaient que la relation qui se manifeste par des dons matériels des premiers contre attente de services des seconds échouait souvent, car le don n'était pas reconnu comme tel par les récipiendaires. Il est donc nécessaire ici, de tenter d'analyser ce qu'entrepreneurs et paysans, employeurs et employés conçoivent être les formes de réciprocité appropriées à leurs relations. Si ces dernières, on l'a vu, s'avèrent avoir été «nouvelles » pour les paysans quand ces derniers passèrent au travail salarié, elles engagent en fait, dans un contexte de marché relativement nouveau au Laos, des formes plus anciennes de comportements, qui sont culturellement comprises par tous comme essentielles. 


\section{Bons et mauvais patrons : celui qui « mange » (kin) et celui qui « nourrit » (lieng)}

Un point récurrent des échanges que j' ai pu avoir avec les entrepreneurs chinois du district était le fait qu'ils tournaient rapidement court. Souvent, en partance pour quelque part au moment où j'arrivais à les rencontrer ou de retour, mais déjà en train pour une autre activité, ils finissaient par s'excuser en reconnaissant qu'ils « n'avaient pas le temps » de prolonger nos entretiens. Dès lors, les conversations se prolongeaient éventuellement avec leurs contremaîtres, mais seulement s'il s'agissait de Laotiens, et rarement avec les ouvriers employés, ces derniers étant également tenus par le temps ; «on aimerait bien faire une pause, mais on est payé à la tâche, cela prend beaucoup plus de temps que prévu de mettre ces haricots en caisse », reconnaissait l'un ; « je vous raconte cela [sur les conditions d'embauche], mais je ne peux pas trop continuer ; ici, il ne faut pas prendre son temps, et je vois le contremaître chinois qui nous regarde », disait une autre ; « comment on peut se reposer un peu, alors qu'ils courent tous ici ? », déplorait un troisième.

Cette attitude tranche singulièrement avec celle d'autres employeurs laotiens, qu'ils soient de petits chefs d'entreprise, des commerçants ou des fonctionnaires du district. Lorsque nous arrivions sur le pas de la porte de leurs maisons, tous étaient affairés, ou affectaient de l'être: «ah, vous avez de la chance de me trouver, normalement, je ne suis jamais à la maison ». La discussion s'amorçait, non sans que tous ne rappellent plusieurs fois leur très grande activité : «je ne m'arrête jamais », « je ne m'assois jamais plus de cinq minutes », « tous les jours, je suis levée vers 6 heures et je ne m'arrête qu'à la nuit tombée ». Pourtant, les entretiens duraient souvent beaucoup plus d'une heure. Face à l'expression de ma gêne de déranger quelqu'un qui se présentait comme aussi affairé, mon hôte ajoutait à ma confusion en démontrant ostensiblement qu'il me donnait, outre des réponses à mes questions, ce qu'il avait de plus précieux : son temps. Il en offrait des démonstrations éclatantes : temps pris à faire chauffer l'eau, servir le thé, le tout sur de longs échanges sur la famille, les enfants, les études, l'embellissement de la maison et le coût des travaux, etc.

Comme avec l'interlocuteur que j'étais, prendre le temps et donner de son temps apparaît comme l'une des principales formes d'obligation réciproque (égale ou inégale, selon la façon dont elle est mise en scène) dans les relations entre salariés et entrepreneurs. Phong, employé comme gardien du nouvel hôtel flambant neuf d'un ancien haut fonctionnaire de la province est moins bien payé qu'un ouvrier agricole dans une plantation. Ceci se vérifie en faisant un calcul économique « rationnel », c'est-à-dire en comparant le nombre d'heures ou de jours où il est immobilisé sur son lieu de travail et qu'il ne peut donc consacrer à ses propres activités, avec le salaire qui lui est versé à la fin du mois. Mais ce n'est pas de cette façon que Phong apprécie ses conditions de travail. Comme pour de nombreux employés interrogés, il ne met pas en avant l'importance du salaire versé lorsqu'il distingue les «bons patrons » des mauvais. Le bon patron, me 
dit-il, même s'il paie moins est celui qui laisse le temps de faire des pauses, en distribuant ou faisant distribuer des cigarettes et de l'alcool par exemple. C'est celui qui consacre du temps à ses employés et qui, comme me l'ont souvent répété de nombreux paysans, les «nourrit » (lieng). Le terme lieng a récemment été associé au pouvoir par les anthropologues [Van Esterik, 1996 ; Evans, 2002] : dans les institutions de l'État, un homme important (phou gnay) doit «nourrir » son réseau familial et les personnes qui lui ont été fidèles, c'est-à-dire leur apporter aide et assistance en cas de besoin (mariages, funérailles, santé, éducation, déplacements, etc.) en échange de leur soutien et de leur aide pour différentes actions. A. Doré [1972, p. 19] précise pourtant, dans l'étude des groupes familiaux de la région de Luang Prabang, que «donner à manger» (lieng) implique forcément une contrepartie : les parents nourrissent leurs enfants en attendant d'être nourris à leur tour par ces derniers dans leurs vieux jours, les animaux sont nourris dans l'expectation d'un bénéfice, les esprits ( $p h i)$ sont nourris dans l'attente de bienfaits dispensés aux humains. Le terme a donc un sens éminemment relationnel, engageant la réciprocité. Mais, nourrir quelqu'un, c'est toujours l'obliger ; et comme le souligne Richard Pottier ${ }^{8}$, lieng en sous-tendant le sens «d'amadouer», « d'appâter », implique un lien de subordination.

Dans le contexte des relations contractuelles entre employeurs et travailleurs, le terme lieng, et le sentiment d'obligation, s'instaurent à travers le temps. Un bon patron, et donc un bon emploi, est celui qui permet à ses employés de profiter de pauses. Me rendant pour travailler et accompagner à deux reprises un même groupe de paysans dans la collecte des haricots, nous prîmes à chaque fois un repas en milieu de journée. Dans le premier cas, les paysans considérèrent qu'ils avaient été mal nourris par le patron, en l'occurrence un Chinois. Dans les faits, ce n'était pas la quantité ni même la qualité de la nourriture qui étaient mises en cause, mais le fait que le repas, apporté tout prêt à la pause par les soins d'un contremaître, n'avait nécessité aucune préparation de la part des employés. De ce fait, nous nous étions tout de suite mis à manger (une opération qui est généralement extrêmement rapide au Laos, les conversations étant rares lorsque l'on mange). Après les quelques cigarettes d'usage en fin de repas, nous nous remîmes à travailler. Le repas du lendemain fut qualifié de meilleur. Pourtant, lors de ce second repas, seul le riz, servi, prêt emballé, dans des sacs plastiques, fut distribué par l'employeur à tout le monde. Il avait donc fallu s'occuper de la confection du repas et nous avions procédé à la pause de la même manière qu'autrefois dans les villages de montagne. L'un était parti chercher de l'eau, un autre des légumes, etc. Durant la cuisson, tout le monde s'assit autour d'un foyer rapidement improvisé et devisa sur des opérations de la matinée ou d'autres sujets. Le temps du repas fut donc beaucoup plus long que le précédent ; aussi fut-il qualifié de «meilleur ». Dans les deux cas, nous avons reçu notre salaire à la fin de la journée, à la tombée de la nuit : 50000 kips par personne le premier jour, 40000 kips le second (soit environ cinq et quatre euros). Pourtant, le groupe fut unanime pour

9. Op. cit. in Doré [1972, p. 21]. 
déclarer ne pas avoir apprécié le premier jour de travail, mais aimé le second, et qualifia ce dernier de «bien » (mouane) en opposition au premier.

Cette situation est bien comprise de nombreux entrepreneurs laotiens. Certains m'expliquèrent comment ils arrivaient à recruter, dans une situation où la maind'œuvre était rare, en mettant en avant, non l'accès à des conditions de travail ou un salaire meilleurs que ceux de leurs concurrents, mais des qualités propres relevant d'une éthique du comportement de l'employeur, à savoir, la générosité et le souci de ses employés. Cette attention passe, on l'a vu, par le don de nourriture : « Pour trouver des journaliers, c'est facile ; ce n'est pas seulement le salaire qui compte, il faut aussi acheter de l'eau, des cigarettes ; c'est comme cela qu'on attire des gens, car les gens ont une bouche et un ventre qui vont avec eux ( $m i$ sop mi thong thi pay nam)». Elle peut aussi se manifester par d'autres types de dons matériels, on l'a vu avec l'entrepreneur racontant comment il avait infléchi la décision de villageois ne voulant pas planter des haricots en dotant leur village d'une maison collective neuve - , mais qui ne suffisent pas.

L'écoute et surtout la parole sont largement valorisées, l'importance de la parole du supérieur hiérarchique étant une donnée ancienne de la culture laotienne ${ }^{9}$. Un commerçant m'expliquait : «Pour faire du commerce, il faut être généreux. Si on n'a pas d'argent pour l'être, il faut utiliser sa bouche et parler aux gens avec amabilité (vаои mouane) ». Il concluait : "En faisant cela, on leur montre qu'avant de parler travail, on passe du temps ensemble ». Khamlek, un entrepreneur local qui me racontait son parcours, insistait sur un point : «Pour réussir, un commerçant doit bien recevoir les gens, sourire, leur donner à manger et à boire. Ceux qui ne font pas cela ne peuvent démarrer aucune affaire. Quand les Akha descendent en ville, certains ne leur donnent rien, mais moi, je leur achète une pipe à eau et du tabac, je les nourris. Ma femme et moi savons un peu de akha et nous communiquons avec eux. J'ai toujours fait cela. Quand d'autres ne pouvaient pas vendre, moi, j'ai toujours pu ». Un autre glosait encore plus explicitement: «Si tu ne sais pas parler, tu as beau être riche, les gens ne te respecteront pas (napteu) ». De l'autre côté, les paysans mettent également en avant l'importance de la parole dans la recherche du travail journalier : « Trouver du travail, cela ne dépend pas du réseau de parents, cela dépend de notre façon de parler »; «Il ne faut pas être taiseux ni être intimidé : il faut parler »; « Trouver du travail, cela dépend de la façon de parler, cela dépend de la face ». On retrouve, dans ces propos, la nécessaire réciprocité dans l'interaction : face à l'employeur, ou à l'entrepreneur, le paysan doit pouvoir maîtriser les codes nécessaires à l'engagement de la relation; le contraire entraîne la honte ou la perte de face.

«Bien parler », c'est aussi utiliser des appellatifs de parenté pour désigner ses employés. Khamlek, de l'ethnie phounoy parle des paysans de l'ethnie akha comme de ses «parents » (pinong); la tenancière de l'hôtel prend chez elle des

10. Louis Finot [1917, p. 140] notait que le traité politique laotien Rājasavan̄̄ définissait l'affabilité ainsi que la parole aimable comme l'un des quatre éléments de popularité et des cinq vertus du souverain. 
«petits-enfants » (luk lane) qui n'ont pourtant aucun lien de parenté avec elle. La réciproque est vraie: Seng se montre fier que ses employés l'appellent tous « Grand frère Seng », car il estime avoir ainsi remporté leur confiance (sua man). $\mathrm{Si}$ ces appellatifs permettent de marquer une proximité entre employeurs et employés, il ne s'agit pas tant d'égalité dans les rapports (les employés Akha de Khamlek continuent de lui témoigner respect et marques de reconnaissance) et certains avouent ne pouvoir faire réellement confiance qu'à leurs véritables parents. Il reste que ces marques verbales de proximité permettent aux entrepreneurs de faciliter leurs affaires. Un commerçant qui achète des produits forestiers aux paysans pour les revendre en Chine m'expliquait: « Si tu ne sais pas bien parler, si tu es radin, les paysans ne viennent pas à toi. Car, au final, notre argent est avec les paysans. Si on sait leur parler, leur argent viendra à nous ».

Ces formes de générosité, de proximité, d'attentions et de confiance s'expriment généralement par les termes de «face» $(n a)$ et de «cœur» (tjay). Seng, par exemple, raconte : «Lorsque j'ai commencé mon activité de commerçant, je n'avais pas d'argent pour offrir à manger quand les paysans venaient chez moi. Mais, dès qu'ils arrivaient, j'achetais de l'eau et leur offrais à boire. Car il faut "présenter tout d'abord son visage et ses yeux" (aou na aou ta kone), c'est-à-dire leur faire un bon accueil ». D'autres insistent sur l'impression de franchise, d'honnêteté, de transparence qu'il faut donner : «Je veux qu'ils voient mon cœur »; «Il faut être généreux ». Et inversement, ne pas faire ce bon accueil serait considéré, dans une certaine mesure, comme une perte de face pour les demandeurs d'emploi. "Ya sou lang kièt», expliquait Taovieng, entrepreneur et chef du plus récent village de la bourgade, formule qu'il glosait de la façon suivante : «Il ne faut pas être méprisant, faire un tri entre les gens et ne parler qu'à ceux qui sont riches ».

C'est donc à la mise en scène de la sociabilité et aux rencontres qu'elle induit que se mesure le prestige d'un entrepreneur ou d'un chef. «Certaines personnes sont des maîtres (tjao), des chefs (nay), mais personne ne vient les voir. D'autres ne le sont pas, mais des gens de partout viennent leur rendre visite. C'est cela être quelqu'un d'important ». Entrer au service de quelqu'un, « se mettre à sa merci » implique qu'en échange l'employeur ou l'entrepreneur compense la perte potentielle de face que cela pourrait représenter pour l'employé par différentes façons de l'appâter (lieng), de nourrir la réciprocité dans la relation, que ce soit par des dons d'objets, de nourriture ou de termes familièrement affectueux.

Autrement dit, « nourrir » (lieng) la relation, c'est aussi établir les formes d'une relation horizontale, telles que la connaissaient les paysans. C'est à ces conditions qu'un travail peut apparaitre «bien » ou «bon » à un paysan, et qu'un employeur peut constituer et maintenir son réseau d'employés journaliers. C'est à ces conditions que les employeurs laotiens se permettent d'affirmer, en désignant leurs concurrents chinois, «nous, on ne "mange" (kin) pas la force de travail des autres ». Dans le contexte traditionnel des relations familiales et villageoises au Laos, Doré [1972, p. 20] soulignait déjà que « manger » (kin) s’opposait fortement 
à «nourrir » lieng, car le terme suggère d'emblée un rapport agonistique et une relation horizontale : «manger » chez quelqu'un, c'est potentiellement s'endetter auprès de cette personne ; de même, l'expression "les parents "mangent" la force de leurs enfants» signifie que les enfants paient leur dette à l'égard de leurs parents en les prenant en charge à leur tour, et met l'accent sur la relation horizontale entre aînés et cadets. Tout l'enjeu entre employeur et employé est donc de faire oublier la verticalité de la relation et de donner à cette dernière les formes de l'horizontalité, par la réciprocité.

Ces règles ne sont pourtant pas mises en pratique par tous les employeurs de la même manière ou avec la même intensité («tout le monde est différent, c'est comme les doigts d'une main »); d'où le fait, au final, que le nombre d'employeurs jugés «bien» par les paysans s'avère relativement restreint. Et que ceux qui n'adoptent pas cette éthique du comportement peinent, jusqu'à présent, à trouver des employés.

\section{Conclusion}

Dans ce passage de la paysannerie au travail salarié, on observe la transformation même de ce qui était pensé comme un travail (asip). L'agriculture d'autosuffisance qui était jusqu'il y a peu la principale activité, et même la seule activité envisageable, en dehors des quelques postes de fonctionnaires, n'est plus vraiment considérée comme un travail, du fait qu'elle ne génère pas de revenus salariés. Ceci a pour conséquence la très importante migration de paysans vers les bourgs, ainsi que le développement de l'emploi journalier. Mais ce changement n'implique pas une totale marchandisation du travail. Le travail reste conçu comme étant aussi une relation sociale, qui implique une certaine coopération entre employeur et employé. Un employé qui met sa force de travail à la disposition d'un employeur estime, implicitement, qu'il met autre chose en jeu que sa force de travail, aussi attend-il d'autres choses en retour que le seul salaire.

Un employeur qui souhaite attirer à lui employés ou «clients » doit donc pouvoir, en plus d'offrir un salaire, consacrer du temps et faire preuve de générosité. Bien sûr, ce type de coopération qui implique plusieurs formes d'échanges entre les partenaires est inégal. In fine, les démonstrations ostentatoires de générosité déployées par les employeurs, qui sont de fait le plus souvent non matérielles, aboutissent à placer l'employeur en position ultime de donneur, mettant implicitement en position d'obligations ses employés. En cela, les nouveaux entrepreneurs dans le nord du Laos se fondent sur des comportements, des gestes qui étaient ceux des élites pré-1975 et sont devenus ceux de cadres du Parti et de hauts fonctionnaires [Evans 2002, p. 105-106].

Faut-il alors suivre J. Roitman [2000, p. 52] qui estime que les rapports sociohistoriques locaux anciens sont inhérents à l'universalisation des modes de pensée capitalistes? En ce sens, les nouveaux entrepreneurs au Laos seraient des 
« médiateurs de la modernité » [ibid.], qui réactualiseraient une éthique traditionnelle dans le contexte économique contemporain.

Mais, on peut également se demander s'il s'agit de formes transitoires d'autorité, dans un monde du travail qui s'avérera de plus en plus dominé par des logiques de marché. Dans le cadre de la future Asean economic community, la libre circulation des travailleurs à travers tous les pays d'Asie du Sud-Est entrera en vigueur. Les Laotiens connaissent pour l'instant mal les modalités de cette libre circulation et la redoutent, imaginant des flux concurrentiels de travailleurs chinois et vietnamiens, qui eux, feront fi des critères qui constituent un «bon» travail et accepteront n'importe quel emploi à n'importe quelle condition.

\section{Bibliographie}

BAIRD I. [2010], «Land, rubber and people : rapid agrarian change and responses in southern Laos », Journal of lao studies, $\mathrm{n}^{\circ}$ 1, p. 1-47.

Bernal V. [1994], «Peasants, capitalism, and (ir)rationality », American ethnologist, $\mathrm{n}^{\circ} 21$, p. 792-810.

BoutÉ V. [2014], « Population's mobility in northern laotian transborder areas », in FAU N., Khonthapane S., TAILlard C. (dir.), Transnational dynamics and territorial redefinitions in southeast Asia : the greater Mekong subregion and Malacca strait economic corridors, Singapour, Institute of southeast asian studies, p. 397-418.

DORÉ A. [1972], De l'hibiscus à la frangipane. Essai de sociologie politique sur la notion d'autorité traditionnelle au Laos, doctorat de $3^{\mathrm{e}}$ cycle, Paris.

DWYER M. [2007], Turning land into capital : a review of recent research on land concessions for investment in Lao PDR, Consultancy report for the Land issues working group, Vientiane : http://rightslinklao.org/wp-content/uploads/downloads/2014/05/Turning-Land-intoCapital-A-review-of-recent-research-on-land-concessions-for-investment-in-Lao-PDRPart-1-of-2-Existing-literature-photo.pdf (page consultée le 10 janvier 2017).

ESTERIK P. (VAN) [1996], "Nurturance and reciprocity in thai studies », in DURRENBERGER P. (dir.), State power and culture in Thailand, New Haven, Yale university, Southeast asian studies p. 22-46.

Evans G. [1990], Lao peasants under socialism, New Haven (Conn.), Yale University press.

Evans G. [2002], A Short history of Laos, Chiang Mai, Silkworm books.

ÉVrard O., BAIRD I. [2017], «The Political ecology of upland/lowland relationships in Laos since 1975 », in Bouté V., PHOLSENA V. (dir.), Changing lives. New perspectives on society, politics, and culture in Laos, Singapore, NUS press, p. 163-191.

FinOT L. [1917], «Recherches sur la littérature laotienne », Bulletin de l'École française d'Extrême-Orient, $\mathrm{n}^{\circ} 17$, p. 1-218.

GoudinEAU Y. (dir.) [1997], Resettlement and social characteristics of new villages : basic needs for resettled communities in the Lao PDR, Vientiane, Unesco/UNDP/Orstom.

HIGH H. [2008], Fields of desire : policy and poverty in Laos, Singapore, NUS press. 
Li Y., LyttLeton C. [2017], "Rubber's affective economies : seeding a social landscape in northwest Laos », in Bouté V., Pholsena V. (dir.), Changing lives. New perspectives on society, politics, and culture in Laos, Singapore, NUS press, p. 301-324.

MARIANI L. [2012], Les avatars du don dans le Laos contemporain. Essai d'anthropologie politique, Paris, Connaissances et savoirs.

ReInHORn M. [1970], Dictionnaire laotien-français, Paris, CNRS.

RIGG J. [2005], Living with transition in Laos market integration in southeast Asia, Londres/New York, Routledge.

Roitman J. [2000], «Économie morale, subjectivité et politique », Critique internationale, $\mathrm{n}^{\circ} 6$, p. 48-56.

Scotт J. [1976], The Moral economy of the peasant. Rebellion and subsistence in South-East Asia, New Haven, Yale university press.

STEERING COMMITTEE FOR CENSUS OF POPULATION AND HOUSING [2006], Results from the population and housing census 2005, Vientiane, ministry of Planning and Investment.

STEERING COMMITTEE FOR CENSUS OF POPULATION AND HOUSING [2016], Results from the population and housing census 2015, Vientiane, ministry of Planning and Investment.

THOMPSON E. P. [1971], « The moral economy of the english crowd in the eighteenth century », Past \& Present, $\mathrm{n}^{\circ}$ 50, p. 76-136. 